\title{
Carcinoma ex Eccrine Spiradenoma
}

National Cancer Institute

\section{Source}

National Cancer Institute. Carcinoma ex Eccrine Spiradenoma. NCI Thesaurus. Code C43344.

A carcinoma that arises in an eccrine spiradenoma. 